\title{
El acceso a las TIC en el hogar y en la escuela: su impacto sobre los logros educativos*
}

\author{
Recibido: 10 de febrero de 2016 - Aceptado: 6 de diciembre de 2016 \\ Doi: dx.doi.org/10.12804/revistas.urosario.edu.co/economia/a.5626
}

\section{María Verónica Alderete ${ }^{\dagger}$ \\ María Marta Formichella ${ }^{\ddagger}$}

\section{Resumen}

El objetivo de este trabajo consiste en analizar la incidencia del acceso a las Tecnologías de la Información y de la Comunicación, tanto en el hogar como en la escuela, sobre el rendimiento educativo de los estudiantes de nivel medio. Para ello, se emplean datos del PISA 2012 para la Argentina. De esta manera, mediante el desarrollo de un modelo multinivel, donde la variable dependiente es el rendimiento educativo en la prueba de matemáticas, se encuentra que el acceso a Computadoras e Internet en el hogar y un mayor número de computadoras por estudiante en la escuela influyen positiva y significativamente en el desempeño educativo.

Palabras clave: Tecnologías de la Información y de la Comunicación, rendimiento educativo, educación media.

Clasificación JEL: I2, O3

* Las autoras agradecen al Proyecto de Desarrollo Tecnológico y Social (PDTS) “Diseño de estrategias para mejorar las oportunidades educativas de la población vulnerable de Bahía Blanca a través de la ONG Red de Voluntarios" y al proyecto "Identificación de políticas educativas eficaces en la mejora de la calidad educativa".

† IIESS, UNS-CONICET; Departamento de Economía, UNS.

‡ IIESS, UNS-CONICET; Departamento de Economía, UNS. Correspondencia: San Andrés 800, Altos de Palihue. Bahía Blanca, Argentina. (C.P. 8000). Tel: +54 (0291) 4595138, Interno: 2700. Fax: +54 (0291) 4595139. Correo electrónico: mformichella@iiess-conicet.gob.ar

Para citar este artículo: Alderete, M. V., \& Formichella, M. M. (2016). El acceso a las TIC en el hogar y en la escuela: su impacto sobre los logros educativos. Revista de Economía del Rosario, 19(2), 221-242.

Doi: dx.doi.org/10.12804/revistas.urosario.edu.co/economia/a.5626 


\title{
Access to ICTs at Home and at School: Impact on Educational Achievements
}

\begin{abstract}
The objective of this paper consists in analysing the incidence of Information and Communication Technologies access at home and at school on the educational performance of intermediate level students. To achieve this goal, data from PISA corresponding to the 2012 year for Argentine is employed. By means of a multilevel model, where the dependent variable is the educational performance in the Maths test, it is found that both access to computers and internet and computers per student at school have a positive and significant effect.
\end{abstract}

Keywords: Information and Communication Technologies, educational achievement, secondary school.

JEL Classification: $\mathrm{I} 2, \mathrm{O} 3$

\section{O acesso às TIC no lar e na escola: o seu impacto sobre os resultados educativos}

Resumo

O objetivo do trabalho consiste em analisar a incidência do acesso às Tecnologias da Informação e da Comunicação, tanto no lar quanto na escola, sobre o rendimento educativo dos estudantes de nível médio. Para isso, empregam-se dados de PISA 2012 para a Argentina. Mediante o desenvolvimento de um modelo multinível, onde a variável dependente é o rendimento educativo na prova de Matemáticas, encontra-se que o acesso a Computadores e Internet no lar e um maior número de computadores por estudante na escola influem positiva e significativamente no desempenho educativo.

Palavras-chave: Tecnologias da Informação e da Comunicação, rendimento educativo, educação média.

Clasificación JEL: I2, O3

\section{Introducción}

Las nuevas Tecnologías de la Información y de la Comunicación (TIC) permiten el desarrollo de nuevas oportunidades de aprendizaje e inclusión de los estudiantes, tanto en el interior de las instituciones educativas como fuera de ellas (Becta, 2007). Asimismo, la educación constituye una prioridad para el desarrollo con equidad en la Sociedad de la Información (Sunkel \& Trucco, 2010).

En este sentido, estas tecnologías representan un importante instrumento para fomentar las prácticas inclusivas (Claro, 2011). Según el proyecto de la CEPAL @LIS2 "Alianza para la Sociedad de la Información 2 - Diálogo político inclusivo e intercambio de experiencias," las TIC no son un fin en sí mismo 
sino solo un medio para alcanzar los objetivos del desarrollo, y en el ámbito escolar, para lograr objetivos educacionales.

El desempeño educativo de los estudiantes está determinado por diversos factores exógenos y endógenos al individuo que interactúan entre sí e involucran los ámbitos sociales, familiares y escolares. En este sentido, las TIC, como la computadora, la telefonía o el acceso a Internet, entre otras, facilitan a los agentes mantenerse conectados entre sí y con el mundo, sin importar la distancia ni el tiempo, y pueden contribuir a mejorar los resultados educativos.

En el sector educativo, el acceso comprende la disponibilidad de recursos tecnológicos de comunicación e información en el establecimiento de enseñanza (Sunkel \& Trucco, 2012). Entre las TIC más básicas, se encuentran las computadoras, las cuales reducen tanto el tiempo como los costos en el proceso educativo (Parshall et al., 2002; Terzis \& Economides, 2011; Witte \& Rogge, 2014). Las computadoras permiten medir y monitorear el aprendizaje de los estudiantes por medio de la recolección de los puntajes obtenidos en los exámenes y, junto con Internet, también facilitan el aprendizaje a distancia (Maguire \& Zhang, 2007). Asimismo, las computadoras e Internet les permiten a los estudiantes hacer las tareas escolares, buscar información por la web, realizar presentaciones, aprender y comunicarse con sus compañeros de clase, entre otras actividades.

Por otra parte, el uso de las TIC está asociado con las capacidades y desarrollo de habilidades para lograr un mejor aprovechamiento de las tecnologías en la educación. De esta manera, se accede de forma más rápida y más eficiente a la información y se reduce su grado de obsolescencia (Lara y Duart, 2005).

Además, las TIC también han mejorado la comunicación entre los distintos agentes del proceso enseñanza-aprendizaje, en especial entre estudiantes y docentes, lo que incrementa los flujos de información y la colaboración entre ellos, más allá de los límites físicos y académicos de las instituciones de enseñanza (Botello \& Rincón, 2012).

Por último, cabe mencionar que, en gran parte, el uso eficaz de las TIC para la educación depende de la actitud de la dirección y de los docentes de los establecimientos educativos para garantizar que el acceso esté acompañado de un uso adecuado. Es decir, el mismo nivel de infraestructura de TIC y la intensidad de su uso puede generar distintos resultados educativos debido a la interrelación de varios factores, entre ellos, el grado de confidencia, la familiaridad de los docentes, estudiantes y padres con las TIC, el acceso a los recursos TIC en el hogar, la escuela o cualquier otro entorno relevante, los efectos entre pares, etc. Por tal motivo, los sistemas educativos en América Latina han trabajado tanto en ofrecer a las escuelas una infraestructura tecnológica adecuada (especialmente computadoras e Internet) como en alfabetizar a los 
estudiantes y profesores en algunos usos básicos de estas (Sunkel, Trucco \& Möller, 2011).

La mayoría de las investigaciones han mostrado relativamente menos atención a las TIC en el hogar, en comparación con el interés de estas en la escuela, pese a que la literatura existente evidencia un uso más frecuente en el hogar, e incluso más preponderante. Sin embargo, ambos recursos resultan complementarios para mejorar el desempeño educativo de los estudiantes.

En tal sentido, el objetivo de este trabajo consiste en extender la literatura acerca del aporte de las TIC al rendimiento educativo de los estudiantes de nivel medio, en el hogar como en la escuela. Con este fin, se estima un modelo Multinivel con datos de PISA correspondientes al año 2012 para la Argentina. Este país es un caso de estudio interesante debido a la puesta en marcha del Programa Conectar Igualdad en el año 2010, el cual facilitó el acceso a las TIC tanto en el hogar como en las escuelas y ha tenido un efecto positivo sobre el rendimiento escolar (Alderete \& Formichella, 2016).

El trabajo se organiza así: en la siguiente sección se presenta la revisión de la literatura, en la sección dos se exhiben la metodología, los datos y las variables; en la tres, se exponen los resultados encontrados y, finalmente, en la sección cuatro se da paso a las conclusiones del trabajo.

\section{Revisión de la literatura}

Para analizar la relación específica entre TIC y rendimiento escolar cabe hacer referencia al estudio de los determinantes del rendimiento educativo. Este tuvo origen en la década del sesenta mediante el informe Coleman (Coleman et al., 1966) y, desde entonces, una gran cantidad de investigaciones se han dedicado a analizar los factores personales, familiares y escolares que inciden en los logros educativos (Calero \& Escardibul, 2007; Calero et al., 2010; Cervini, 2002, 2003, 2012; Formichella, 2011; Formichella \& Krüger, 2013). Entre ellos, algunos estudios han introducido a las TIC como variable de control (Decándido, 2011; Formichella \& Krüger, 2013; Santos, 2007), pero no las han incorporado entre los principales determinantes del logro educativo.

En la Argentina son escasos los trabajos que se han focalizado en las TIC como factor explicativo del rendimiento educativo (Alderete \& Formichella, 2015; Formichella et al., 2015). Sin embargo, el efecto de las TIC en el rendimiento educativo ha sido estudiado por varios autores considerando otros ámbitos geográficos (Aristazabal et al., 2009; Botello \& Rincón, 2014; Buckingham, 2008; Carrillo et al., 2010; Comber et al., 2002; Cristia et al., 2010, 2012; Cox et al., 2004; Kuhlemeier \& Hemker, 2007; Machin et al., 2006; Malamud \& 
Popeleches, 2010; Mediavilla \& Escardíbul, 2015; Notten \& Kraaykamp, 2009; Spieza, 2010). La mayoría de las investigaciones han abordado el impacto de las TIC tanto en el hogar como en la escuela sobre los resultados educativos, con algunas excepciones que se centran únicamente en las TIC en el hogar (Jewitt \& Parashart, 2011; Kuhlemeier \& Hemker, 2007; Notten \& Kraaykamp, 2009).

Las TIC han transformado los procesos de enseñanza y aprendizaje y mejorado las estrategias para el logro educativo (Carneiro et al., 2009; Kozma, 2003, 2008; Rodríguez et al., 2013; Sunkel, 2006). Asimismo, los instrumentos y herramientas utilizadas dentro y fuera del contexto educativo son medios significativos para mejorar el desempeño de los infantes (Aypay, 2010; Biagi \& Loi, 2013; Meelissen \& Drent, 2008).

El aprovechamiento de las TIC por parte de un estudiante no solo depende de las oportunidades de acceso disponibles, sino también del tipo de actividad realizada mediante las nuevas tecnologías. A diferencia de la primera brecha digital que se refiere a las diferencias de acceso, existe una segunda brecha digital relativa a las diferencias en el uso de las TIC y a la capacidad de beneficiarse de ellas (Hargittai, 2002; Robinson, DiMaggio \& Hargittai, 2003). En este sentido, el acceso a las TIC en el hogar cumple un rol importante para el desarrollo de las habilidades digitales requeridas en la escuela (Kulhemier \& Kemker, 2007).

No solo interesa haber nacido con posibilidad de acceso a las TIC y al entorno digital en el hogar, sino que efectivamente importa la presencia de un ambiente familiar con el know-how o el conocimiento del lenguaje digital, así como la capacidad y disposición para apoyar al niño a dar sus "primeros pasos" en el mundo virtual (Buckingham, 2008). Las habilidades digitales o ICT skills son parte de los objetivos educacionales, ya que estas contribuyen a obtener mejores resultados en la escuela y un mejor desempeño (Kulhemier \& Kemker, 2007).

Teniendo en cuenta que existe una fuerte asociación entre educación e ingresos, cuanto más alto es el nivel educativo de los padres, es mayor la probabilidad de que los hijos dispongan en sus hogares de equipos y conexiones a Internet (Notten et al., 2009; Buckingham, 2008); que los adultos del hogar conozcan y sepan utilizar las herramientas digitales y puedan enseñar y transmitir tales nociones y pericias, así como motivar a sus hijos. A su vez, también será más probable el acceso a las redes sociales que los motiven a vincularse con la tecnología y que confieran sentido a su uso. Cuanto más pobres los hogares, es más probable que los padres no dispongan de suficiente calificación para un mejor aprovechamiento de las TIC. Esto termina redundando en el desarrollo, no solo de mundos digitales, sino también sociales diferentes.

El informe publicado de PISA TIC 2006 (OECD, 2009) encuentra una correlación positiva entre las TIC en el hogar y los logros educativos, aun controlando 
por nivel socioeconómico; en contraste, no hallan una correlación significativa con las TIC en el centro educativo.

Spiezia (2010) analiza el impacto de las TIC sobre los resultados educativos medios con el programa PISA 2006 para el total de los países participantes y destaca el rol del uso de estas tecnologías en los hogares de los estudiantes. El autor encuentra un efecto significativo y positivo del uso de las computadoras en el hogar sobre el rendimiento escolar, cuyo impacto es superior al obtenido por el uso en las escuelas.

Aristazabal et al. (2009) analizan los factores que inciden sobre los logros escolares, medidos de acuerdo con la prueba PISA 2006 y 2009 en Colombia. Mediante un modelo de ecuaciones estructurales, encuentran que tanto las TIC del hogar (uso de Internet y sus herramientas, y otros dispositivos como consolas de juegos) como las escolares (computadora y software educacional) afectan positivamente el rendimiento de los estudiantes. A diferencia de Spiezia (2010), quien encuentra una contribución mayor de las TIC en la escuela.

Botello y Rincón (2014) hallan que la tenencia de una conexión a Internet en los hogares puede incrementar hasta en un $0,57 \%$ el puntaje promedio de los estudiantes junto con un 3,6\% de la tenencia de PC en la casa, con datos para algunos países de América Latina. Asimismo, los autores encuentran que una mayor proporción del ratio computadoras por alumno en los colegios incrementa en un 0,01\% cada una de las áreas evaluadas en la prueba. En este orden, Notten y Kraaykamp (2009) hallan una correlación positiva y significativa entre la disponibilidad de TIC en el hogar y los puntajes obtenidos en los exámenes PISA en Ciencia.

Por el contrario, algunos autores no encuentran una incidencia significativa de las TIC en el hogar sobre los logros educativos (Malamud \& Popeleches, 2010; Wittwer \& Senkbeil, 2008). Malamud y Popeleches (2010) estiman el efecto de las computadoras en el hogar sobre los logros educativos de los estudiantes en Romania. Los autores aprovechan la discontinuidad de un subsidio destinados a familias de bajos ingresos para evaluar el impacto de las TIC mediante un cuasiexperimento. Según estos autores, los estudiantes que usan el subsidio para comprar una computadora para el hogar obtienen menores puntajes en matemáticas, inglés y rumano.

De igual manera, Wittwer y Senkbeil (2008) no encuentran una influencia sustancial del uso de computadoras en el hogar sobre los puntajes obtenidos en las pruebas PISA de matemáticas para Alemania.

Jewitt y Parashart (2011) evalúan un programa en el Reino Unido que entregaba una computadora con Internet a familias de bajos recursos con hijos entre 5 y 19 años durante un año. Los resultados de la evaluación indican 
que los estudiantes incrementan el tiempo dedicado a las tareas escolares y al aprendizaje independiente con la computadora en el hogar.

Plowman et al. (2011) analizan un programa de acceso a las TIC en el hogar para proveer computadoras e Internet a niños en etapa preescolar. Concluyen que el acceso a la tecnología en el hogar no es el criterio clave o razón suficiente para garantizar su uso. Esto depende de un complejo de actitudes de los padres, de las aspiraciones educacionales de los padres, y cuánta supervisión se requiere y es ofrecida.

Kuhlemeier y Hemker (2007) estudian el impacto del uso de Internet y de la computadora en el hogar sobre las habilidades digitales requeridas en la escuela en estudiantes de nivel secundario de 13 a 15 años en Alemania. Los autores encuentran que el uso en el hogar de las TIC está sustancialmente relacionado con las habilidades digitales en la escuela.

En la Argentina, Alderete y Formichella (2015) encuentran que existen diferencias estadísticamente significativas en el rendimiento educativo promedio, derivadas de la participación en el Programa Conectar Igualdad con datos de PISA correspondientes al año 2012. Asimismo, Formichella et al. (2015) hallan diferencias estadísticamente significativas en el rendimiento educativo promedio derivados del uso de las TIC en el hogar.

\section{Metodología, datos y variables}

\subsection{Metodología}

Cuando a partir de una población se selecciona una muestra por etapas, es decir; cuando en primer lugar se elige a partir de una estructura mayor y luego se seleccionan casos dentro de esta, lo que existe es una estructura multinivel jerárquica y dependencia de las observaciones dentro de cada conjunto (Hox, 1995).

En este sentido, diversos autores han planteado que el caso de las escuelas que agrupan clases, las cuales, a su vez, agrupan alumnos, es un ejemplo típico de estructura multinivel, y han expresado que, por ello, resulta conveniente utilizar modelos de regresión multinivel cuando se estudian los determinantes del rendimiento educativo (Bryk \& Raudenbusch, 1988; Calero et al., 2009; Cervini, 1999; Escardibul \& Mediavilla, 2015; Formichella, 2011; Formichella \& Krüger, 2012; Hox, 1995; Krüger, Formichella \& Lekouna, 2014). Como ya se ha mencionado, en este trabajo se utilizan datos de la prueba PISA, los cuales se recolectan teniendo en cuenta escuelas y, luego, los alumnos dentro de estas. 
Por ello, la metodología escogida para cumplir con el objetivo propuesto es una regresión econométrica de tipo multinivel.

Diez Roux (2002) menciona que la ventaja de utilizar el análisis multinivel es que incorpora la consideración de la dependencia de las observaciones al interior de cada grupo. Como se explica en OCDE (2003), este tipo de regresión considera que las observaciones individuales se encuentran agrupadas en unidades más grandes, es decir, incorpora información extra vinculada con el anidamiento de los datos.

Es así que, a partir de una regresión econométrica de tipo multinivel, se realiza el cálculo de una ecuación por cada grupo y no el de una única ecuación sobre el conjunto total de estos. Esto permite obtener mejores estimadores de los coeficientes de regresión y de su variación, en comparación con los modelos tradicionales representados principalmente por los modelos de Mínimos Cuadrados Ordinarios (De la Cruz, 2008) y posibilita estudiar, al mismo tiempo, los efectos de las variables grupales e individuales sobre los resultados individuales (Diez Roux, 2002).

Además, este tipo de regresión permite que se realicen los test estadísticos estándar con mayor eficiencia. Esto se debe a que parten del supuesto de independencia de las observaciones y, si este es violado (como ocurre en las estructuras multinivel), las estimaciones de los errores estándar de los test estadísticos convencionales son más pequeñas y pueden causar distorsiones en los resultados de dichos test (Hox, 1995).

Finalmente, es preciso señalar que los modelos de tipo multinivel pueden desarrollarse en forma simple o compleja. La primera, supone que las discrepancias entre los grupos, en este caso, entre las escuelas, solo puede observarse en la constante. Esto implica que los efectos de cada variable explicativa sobre la dependiente son fijos entre grupos, es decir, las rectas de regresión de cada colegio son paralelas y solo difieren en la ordenada al origen. Por otra parte, la segunda forma consiste en incorporar diferencias entre los grupos, no solo en el intercepto, sino también en los coeficientes de algunas variables independientes. En este caso, las rectas de regresión difieren en la ordenada al origen y en las pendientes y se dice que el modelo incluye efectos aleatorios. Es importante señalar que el intercepto debe definirse con efectos aleatorios, sino, no tendría sentido aplicar un modelo de tipo multinivel (OCDE, 2003).

Dado que los modelos jerárquicos presentan diferentes categorías, existen variables en cada una de ellas. En el caso propuesto en este trabajo existen dos niveles: escuelas y alumnos; por ello, hay algunas variables que caracterizan a los colegios (nivel 2) y otras a los individuos (nivel 1). Las primeras son iguales para todos los estudiantes que asisten al mismo establecimiento educativo, mientras que las segundas se corresponden con características 
propias de cada alumno. Así, las variables del nivel 2 solo influyen sobre los interceptos de las escuelas (OCDE, 2003).

Por su parte, las variables de nivel 1 pueden ser incorporadas al modelo con efectos fijos o aleatorios, en función de la teoría sobre el tema y/o los objetivos de investigación. En el primer caso, solo influyen sobre la ordenada al origen de cada regresión, mientras que en el segundo también afectan a las pendientes. En este caso, si los efectos aleatorios resultan ser estadísticamente significativos quiere decir que hay discrepancias en lo que las escuelas hacen en relación con las diferencias de origen de quiénes asisten a estas (OCDE, 2003).

Aquí, la variable de nivel 1 TIC se ha incorporado con efectos aleatorios con el fin de establecer si las escuelas difieren en su accionar en relación con las brechas de origen que poseen sus alumnos en cuanto a la dotación de tecnología en sus hogares.

Formalmente, suponiendo que hay un modelo de rendimiento educativo conformado por dos niveles y que incorpora tres variables explicativas, una en el nivel 2 (P) y dos en el nivel 1 ( $\mathrm{X}$ de efectos fijos y $\mathrm{Z}$ de efectos aleatorios); lo explicado en los párrafos anteriores puede expresarse de la siguiente manera:

- Nivel 1:

$$
Y_{i j}=\beta_{0 j}+\beta_{1 j} X_{i j}+\beta_{2 j} Z_{i j}+r_{i j}
$$

- Nivel 2:

$$
\begin{gathered}
\beta_{0 j}=\gamma_{00}+\gamma_{01} P_{j}+\mu_{0 j} \\
\beta_{1 j}=\gamma_{10} \\
\beta_{2 j}=\gamma_{20}+\mu_{2 j}
\end{gathered}
$$

- Modelo completo:

$$
Y_{i j}=\gamma_{00}+\gamma_{01} P_{j}+\gamma_{10} X_{i j}+\gamma_{20} Z_{i j}+\mu_{0 j}+\mu_{2 j} Z_{i j}+r_{i j}
$$

Donde:

- $\mathrm{Y}_{\mathrm{ij}}$ es el resultado educativo (esperado) del alumno " $\mathrm{i}$ " en la escuela " $\mathrm{j}$ ".

- $\quad \beta_{0 \mathrm{j}}$ es el intercepto de la recta de regresión para la escuela " $\mathrm{j}$ ". 
- $\quad \beta_{1 j}$ es el coeficiente que acompaña a la variable explicativa $X$, la cual está incorporada al modelo con efectos fijos, por lo tanto, $\beta_{1 \mathrm{j}}$ es igual para todos los centros y se representa mediante $\gamma_{10}$.

- $\quad \beta_{2 j}$ es el coeficiente que acompaña a la variable explicativa $Z$, la cual está incorporada al modelo con efectos aleatorios, por lo tanto, varía entre centros. Está formada por una parte fija $\left(\gamma_{20}\right)$ y una parte aleatoria $\left(\mu_{2 j}\right)$. Esta última representa la distancia residual desde el coeficiente de regresión de los centros hasta $\gamma_{20}$.

- $\gamma_{01}$ es el coeficiente que acompaña a la variable explicativa P (primera y única del nivel 2, por eso el subíndice es "01"). Dado que pertenece al nivel 2 , existe un valor de P por cada centro " $\mathrm{j}$ ". Como puede observarse en la ecuación, esta variable influye en el valor del intercepto $\beta_{0 j}$.

- $r_{i j}$ es la varianza residual dentro de cada centro.

- $\quad \mu_{0 j}$ es la varianza residual entre centros.

En los análisis multinivel resulta útil estimar un modelo sin incluir variables explicativas, al cual se lo denomina "modelo nulo". Este modelo brinda información sobre qué proporción de la desigualdad en los resultados de rendimiento educativo se debe a diferencias entre grupos y qué proporción se vincula con diferencias al interior de estos. Formalmente, el modelo se expresa así:

$$
Y_{i j}=\gamma_{00}+\mu_{0 j}+r_{i j}
$$

Donde:

- $\gamma_{00}$ representa los efectos fijos o determinísticos (intercepto global).

- $\mu_{0 \mathrm{j}} \mathrm{y}_{\mathrm{ij}}$ representan los efectos aleatorios o estocásticos.

En este caso, los interceptos de cada grupo (los $\beta_{0 j}$ ) son iguales a las medias de los grupos o muy cercanos a estas. Como puede observarse, $\beta_{0 j}$ está formado por una parte fija, común a todos los grupos $\left(\gamma_{00}\right)$ y una parte aleatoria $\left(\mu_{0 j}\right)$, que representa la desviación del grupo " $\mathrm{j}$ " respecto de $\gamma_{00}$. Cabe señalar que $\gamma_{00}$ es el resultado del conjunto de grupos (promedio) y es denominado intercepto global. Entonces, $\mu_{0 j}$ es la desviación del grupo "j" del promedio del conjunto de los grupos y representa la varianza entre estos.

Por otra parte, $\mathrm{r}_{\mathrm{ij}}$ es el desvío del resultado del individuo " $\mathrm{i}$ ", respecto del promedio del grupo " $\mathrm{j}$ " al que pertenece. Puesto que en este modelo a cada individuo se le asigna el promedio de su grupo como resultado predicho, $\mathrm{r}_{\mathrm{ij}}$ es igual a la varianza dentro de cada grupo. 
Entonces, cuando se estudia el rendimiento educativo con base en un modelo nulo, las varianzas residuales intraescuelas e interescuelas son iguales a las estimaciones de las varianzas intragrupos e intergrupos. A partir de esta información, es posible calcular el coeficiente de correlación intraclase ( $\rho)$, el cual representa la proporción de la varianza residual explicada por diferencias entre escuelas $\left(\rho=\mu_{0 j} /\left(\mu_{0 j+} r_{i j}\right)\right.$, es decir, la proporción de la varianza de los resultados educativos entre centros. En este punto, es relevante señalar que, si dicho valor fuera cero no tendría sentido plantear un modelo multinivel (OCDE, 2003).

Con respecto a la bondad de ajuste, es interesante conocer un indicador denominado "varianza explicada", el cual puede obtenerse de comparar el modelo propuesto con el modelo nulo. La manera de calcular este indicador es la siguiente:

Tabla 1. Varianza explicada

\begin{tabular}{ll}
\hline Total & $1-\left(\mu_{0 j+} r_{i j}\right)_{\text {Modelo propuesto }} /\left(\mu_{0 j+} r_{i j}\right)_{\text {Modelo nulo }}$ \\
Nivel 1 (alumnos) & $1-\left(r_{i j}\right)_{\text {Modelo propuesto }} /\left(r_{\mathrm{ij}}\right)_{\text {Modelo nulo }}$ \\
Nivel 2 (escuelas) & $1-\left(\mu_{0 j}\right)_{\text {Modelo propuesto }} /\left(\mu_{0 j}\right)_{\text {Modelo nulo }}$ \\
\hline
\end{tabular}

Fuente: elaboración propia con base en OCDE 2003.

Finalmente, cabe describir la forma general de los modelos econométricos que se utilizan en este trabajo. Como se verá en la próxima sección, no se cuenta con datos a nivel de las clases, por lo que se utilizan modelos jerárquicos de dos niveles: alumnos y escuelas. Asimismo, se permite la variación entre escuelas del término constante y de las pendientes. A continuación se presenta la expresión formal de los modelos:

- Nivel 1

$$
Y_{i j}=\beta_{0 j}+\sum_{k=1}^{K} \gamma_{k 0} X_{k i j}+\sum_{m=1}^{M} \beta_{m j} Z_{m i j}+r_{i j}
$$

- Nivel 2

$$
\begin{gathered}
\beta_{0 j}=\gamma_{00}+\sum_{f=1}^{F} \gamma_{0 f} P_{j}+u_{0 j} \\
\beta_{m j}=\gamma_{m 0}+u_{m j}
\end{gathered}
$$


Donde K es el número de variables explicativas de nivel 1 que se incorporan al modelo con efectos fijos (X); $\mathrm{M}$ es el número de variables explicativas de nivel 1 que se incorporan al modelo con efectos aleatorios $(Z)$ y $F$ el número de variables explicativas de nivel $2(\mathrm{P})$. El resto de la nomenclatura ya ha sido explicada previamente.

Por último, resta mencionar que en este trabajo se utiliza el software STATA.12 para calcular los parámetros de las ecuaciones anteriores, los cuales se estiman simultáneamente mediante métodos iterativos que maximizan una función de máximo verosimilitud.

\subsection{Los datos}

El análisis de regresión multinivel descrito en la sección anterior se realiza con datos provenientes del Programa PISA del año 2012, que es realizado por la Organización para la Cooperación y el Desarrollo Económico (OCDE) y su objetivo es medir las competencias de los estudiantes de 15 años de diferentes países para poder evaluar en qué medida están preparados para enfrentarse a los desafíos que tendrán en su vida adulta viviendo en sociedad. Este programa es llevado a cabo cada tres años y en cada oportunidad se elige un área de evaluación como prioritaria. PISA 2012 hizo hincapié en la competencia matemática (OCDE, 2006).

Cabe aclarar que los resultados de las evaluaciones son presentados utilizando valores plausibles (PV). Estos valores son una representación del conjunto de capacidades de un estudiante. Surgen del hecho de que cada individuo responde solo a cierto número de ítems de la prueba y se vuelve necesario estimar cómo hubiera contestado en la totalidad de los casos. Así, se evita el sesgo que existiría si se predice la habilidad (no observable) en función de un número limitado de datos observables. Para estimar estos valores el equipo de PISA utiliza un software específico (Martinez Arias, 2006; OCDE, 2003).

En este contexto, en OCDE (2003) se explica que los estadísticos poblacionales y los parámetros de los modelos de regresión deben estimarse usando los valores plausibles separadamente; mientras que el valor del estadístico poblacional debe calcularse como el promedio de los estadísticos obtenidos con cada uno de estos (OCDE, 2003).

Al momento de realizar este trabajo, los últimos datos disponibles son los de PISA 2012 y por ello es la base de datos elegida. En la Argentina, en el programa PISA 2012 fueron evaluados 5908 estudiantes de 15 años, quienes asistían a 226 establecimientos educativos diferentes. 


\subsection{Variables}

Las variables utilizadas para estimar los modelos descritos en la metodología se presentan en la tabla 2 junto con sus estadísticos descriptivos.

Tabla 2. Descripción de variables y estadísticos descriptivos

\begin{tabular}{|c|c|c|c|c|c|c|c|}
\hline Variables & Descripción & $\begin{array}{l}\text { Signo es- } \\
\text { perado }\end{array}$ & $\mathrm{N} .^{\circ}$ & Promedio & $\begin{array}{l}\text { Desvío } \\
\text { estándar }\end{array}$ & Mínimo & Máximo \\
\hline \multicolumn{8}{|c|}{ Dependiente } \\
\hline PVMATH & $\begin{array}{l}\text { Puntuación de la } \\
\text { prueba PISA en la } \\
\text { competencia mate- } \\
\text { mática, variable con- } \\
\text { tinua de } 0 \text { a } 100\end{array}$ & & 5908 & 395,6357 & 80,45089 & 51,88502 & 674,86368 \\
\hline \multicolumn{8}{|c|}{ Independientes de Nivel 1} \\
\hline TIC & $\begin{array}{l}\text { Variable binaria, to- } \\
\text { ma valor } 1 \text { si el indi- } \\
\text { viduo tiene computa- } \\
\text { dora con Internet en } \\
\text { el hogar y } 0 \text { en caso } \\
\text { contrario }\end{array}$ & Positivo & 5488 & 0,7530977 & 0,4312487 & 0 & 1 \\
\hline $\begin{array}{l}\text { Repeti- } \\
\text { dor }\end{array}$ & $\begin{array}{l}\text { Toma valor } 1 \text { si el es- } \\
\text { tudiante ha repetido } \\
\text { algún año y } 0 \text { en caso } \\
\text { contrario }\end{array}$ & Negativo & 4761 & 0,231674 & 0,4219462 & 0 & 1 \\
\hline HISEI & $\begin{array}{l}\text { Índice construido por } \\
\text { PISA. Representa el es- } \\
\text { tatus ocupacional de } \\
\text { los padres (considera } \\
\text { el estatus más alto en- } \\
\text { tre padre y madre). }\end{array}$ & $\begin{array}{l}\text { Positivo } \\
\text { (Formi- } \\
\text { chella, } \\
\text { 2011). }\end{array}$ & 5228 & 45,44809 & 22,56358 & 11,01 & 88,96 \\
\hline $\begin{array}{l}\text { Secunda- } \\
\text { rio_ma- } \\
\text { dre }\end{array}$ & $\begin{array}{l}\text { Variable binaria. To- } \\
\text { ma valor } 1 \text { si la ma- } \\
\text { dre del alumno posee } \\
\text { secundario completo } \\
\text { y } 0 \text { en caso contrario. }\end{array}$ & $\begin{array}{l}\text { Positivo } \\
\text { (Formi- } \\
\text { chella, } \\
\text { 2011). }\end{array}$ & 4809 & 0,6082346 & 0,4881955 & 0 & 1 \\
\hline
\end{tabular}

Independientes de Nivel 2

Variable informada por PISA, representa el ratio de computa-

ratcmp15 doras destinadas a educación sobre la $\begin{array}{llllll}\text { Positivo } & 5369 & 0,7601775 & 2,104167 & 0 & 27,5\end{array}$ cantidad de alumnos de 15 años de la escuela. 


\begin{tabular}{|c|c|c|c|c|c|c|c|}
\hline Variables & Descripción & $\begin{array}{l}\text { Signo es- } \\
\text { perado }\end{array}$ & N. ${ }^{\circ}$ & Promedio & $\begin{array}{l}\text { Desvío } \\
\text { estándar }\end{array}$ & Mínimo & Máximo \\
\hline Privada & $\begin{array}{l}\text { Variable binaria. Va- } \\
\text { lor } 1 \text { la escuela es de } \\
\text { gestión privada y } 0 \\
\text { en caso contrario }\end{array}$ & $\begin{array}{l}\text { Positivo } \\
\text { (Formi- } \\
\text { chella, } \\
\text { 2011; } \\
\text { Formi- } \\
\text { chella \& } \\
\text { Krüger, } \\
\text { 2013) }\end{array}$ & 5785 & $0 ; 1695765$ & 0,3752928 & 0 & 1 \\
\hline \multicolumn{8}{|c|}{ Variables de control } \\
\hline Mujer & $\begin{array}{l}\text { Toma valor } 1 \text { para las } \\
\text { estudiantes mujeres y } \\
0 \text { para los estudiantes } \\
\text { varones }\end{array}$ & $\begin{array}{l}\text { Negativo } \\
\text { (Formi- } \\
\text { chella e } \\
\text { Ibañez, } \\
\text { 2014). }\end{array}$ & 5908 & 0,5269127 & 0,4993174 & 0 & 1 \\
\hline
\end{tabular}

Fuente: elaboración propia con base en PISA 2012.

Como ya se ha mencionado, la variable dependiente escogida para estudiar el rendimiento educativo es PVMATH, debido a que ha sido la competencia central en el operativo 2012. De acuerdo con los antecedentes revisados, se espera que las variables TIC y RATCMP15 tengan un efecto estadísticamente significativo y positivo sobre los logros escolares.

\section{Resultados}

En esta sección, se exhiben los resultados encontrados al estimar diferentes modelos de tipo multinivel a partir de la base de datos PISA 2012.

En primer lugar, se estima un modelo nulo con el fin de corroborar la conveniencia de utilizar regresiones de tipo multinivel. Para ello, se consideran dos indicadores: por un lado, la significatividad de los efectos aleatorios de la constante y, por otra parte, el coeficiente de correlación intra-clase $(2695.55 / 3361.59=0.45)$. Dicha información es posible de recoger de la tabla $\mathrm{N}^{\circ} 3 \mathrm{y}$, como puede observarse, los efectos aleatorios de la varianza de la constante son estadísticamente significativos y, asimismo, la proporción de la brecha en los resultados explicada por diferencias entre las escuelas es positiva (0.45).

Luego, se estima un modelo en el que solo se incluyen variables independientes de nivel uno (Modelo 1). A partir de este, se hace hincapié en el signo y la significatividad estadística del coeficiente que acompaña a la variable TIC (tabla 4). El valor de este es 14049 y es significativo al 5\%. Eso quiere decir que las TIC se asocian positivamente con el rendimiento educativo. 
Tabla 3. Estimación de los efectos aleatorios de los parámetros

\begin{tabular}{|c|c|c|c|c|}
\hline & Modelo nulo & Modelo 1 & Modelo 2 & Modelo 3 \\
\hline Varianza de la constante & $2695,55^{* *}$ & $2695.55^{* *}$ & $1188,60^{* *}$ & $1010,55774^{* *}$ \\
\hline Varianza residual & $3361,59 * *$ & $3361.59^{* *}$ & $2860,5468^{* *}$ & $1856,81888^{* *}$ \\
\hline Varianza TIC & & & $228,21^{* *}$ & \\
\hline Varianza (RATCMP15) & & & & $2,30084 \mathrm{E}-07^{* *}$ \\
\hline Varianza (privada) & & & & 529,1470001 \\
\hline
\end{tabular}

**Sig. al $5 \%$.

Fuente: elaboración propia con base en PISA 2012.

Asimismo, con el objeto de estudiar si las escuelas difieren en lo que hacen con las discrepancias de origen que traen los estudiantes en cuanto la disponibilidad de TIC en sus hogares, se estima un modelo igual al anteriormente presentado, con la característica particular de que se le incorporan efectos aleatorios a las variable TIC de nivel 1 (Modelo 2). Como puede observarse en la tabla 4, la variable TIC continúa siendo estadísticamente significativa y, como se exhibe en la tabla 3, sus efectos aleatorios también lo son. Esto implica que efectivamente los centros escolares difieren en su accionar respecto a la brecha tecnológica de sus estudiantes y que, por ende, existen estrategias que los establecimientos educativos pueden aplicar para disminuirla.

Finalmente, se estima un modelo que, además de las variables de nivel 1 con efectos fijos ${ }^{1}$, incorpora variables explicativas en el nivel escuelas (Modelo 3). En este caso, interesa especialmente la variable RATCMP15: importa en qué sentido se relaciona con la varianza del intercepto y su grado de significatividad estadística. Como puede verse en la tabla 3, el signo de la varianza de RATCMP15 es positivo y esta es estadísticamente significativa a un nivel de confianza del $95 \%$. Es decir, los estudiantes que asisten a escuelas que cuentan con mayor disponibilidad de computadoras por alumno obtienen, en promedio, un mayor rendimiento. Además, en este modelo la variable TIC del hogar continúa siendo significativa (al 10\%), aunque el valor de su coeficiente es menor que en los modelos anteriores (tabla 4). Esto puede deberse a que, en los Modelos 1 y 2 propuestos anteriormente el efecto de TIC estaba sobreestimado, parte de este correspondía a la variable RATCMP15, la cual no había sido aún incluida.

1 No se incorporan en este caso efectos aleatorios de la variable TIC porque el modelo en el software no converge. 
Tabla 4. Coeficientes estimados de los Modelos

\begin{tabular}{lccc}
\hline \multicolumn{1}{c}{ Variables } & Modelo 1 & Modelo 2 & Modelo 3 \\
\hline Mujer & $-27,410^{* * *}$ & $-27,635578^{* * *}$ & $-28,3024^{* * *}$ \\
Repetidor & $-44,679^{* * *}$ & $-44.770858^{* * *}$ & $-47,963944^{* * *}$ \\
HISEI & $0,281^{* *}$ & $0,28389182^{* *}$ & $0,3111319^{* *}$ \\
secundario_madre & $13,557^{* *}$ & $13,4846978^{* *}$ & $11,1324276^{* *}$ \\
TIC & $14,049^{* *}$ & $15,043292^{* *}$ & $12,160193^{*}$ \\
_cons & $407,215^{* * *}$ & $406,15368^{* * *}$ & $405,5058^{* * *}$ \\
\hline
\end{tabular}

*, ** $\mathrm{y}^{* * *}$, sig. al 10\%, $5 \%$ y $1 \%$ respectivamente.

Fuente: elaboración propia con base en PISA 2012.

Finalmente, en la tabla 5 se presenta el porcentaje de varianza explicada en cada uno de los modelos estimados, siendo el último presentado (Modelo 3) el que logra una mejor bondad de ajuste, al explicar un $53 \%$ de la discrepancia de los resultados educativos. Cabe señalar que este valor es consistente con la literatura sobre el tema. De este modo, se logra explicar un $63 \%$ de las diferencias originadas por desigualdades a nivel de escuelas y un $45 \%$ de las diferencias generadas por discrepancias entre individuos.

Tabla 5. Porcentaje de varianza explicada

\begin{tabular}{lcccc}
\hline \multirow{2}{*}{ Varianza } & \multicolumn{4}{c}{ PVMATH } \\
\cline { 2 - 5 } & Modelo nulo & Modelo 1 & Modelo 2 & Modelo 3 \\
\hline Varianza entre escuelas & 2696 & 1398,65 & 1188,60 & 1010,55774 \\
Varianza entre alumnos & 3362 & 2876,01 & 2860,55 & 1856,81888 \\
Varianza total & 6057,133 & 4274,6626 & 4049,14488 & 2867,37662 \\
Porcentaje de varianza residual explicado por & & $48 \%$ & $56 \%$ & $63 \%$ \\
las variables sobre el modelo nulo: nivel escuelas & & & $15 \%$ & $45 \%$ \\
$\begin{array}{l}\text { Porcentaje de varianza residual explicado por las } \\
\text { variables sobre el modelo nulo: nivel alumnos }\end{array}$ & & & & \\
$\begin{array}{l}\text { Porcentaje de varianza residual explicado por } \\
\text { las variables sobre el modelo nulo: total }\end{array}$ & $29 \%$ & $33 \%$ & $53 \%$ \\
\hline
\end{tabular}

Fuente: elaboración propia con base en PISA 2012.

\section{Conclusiones}

El objetivo de este trabajo fue analizar la relación de las TIC, tanto en el hogar como en la escuela, con el rendimiento educativo de los estudiantes de nivel 
medio en la Argentina. Mediante el empleo de modelos de tipo multinivel y datos de PISA 2012, correspondientes a la prueba de matemáticas, se concluye que la familia es uno de los agentes fundamentales para, junto con las escuelas, potenciar el aporte de las tecnologías de la información y de la comunicación en la educación.

Este trabajo ha pretendido ir más allá de la simple dicotomía entre acceso a las TIC en el hogar frente al acceso a las TIC en la escuela. Ambos tipos de acceso son recursos que se vinculan con el desempeño educativo, es decir que las tecnologías tanto en el ámbito del hogar y la escuela contribuyen a mejorar la performance de los estudiantes y la evidencia aquí presentada sustenta esta idea. Tanto la variable TIC de nivel uno, como la variable RATCMP15 de nivel dos han resultado ser estadísticamente significativas y su signo positivo. Es decir, tanto la disponibilidad de computadoras con Internet en el hogar como la disponibilidad de computadoras para estudiar en la escuela se asocian con un mayor rendimiento escolar.

Respecto del impacto de las TIC en el hogar sobre el rendimiento educativo, los resultados concuerdan con los estudios existentes (Aristazabal et al, 2009; Botello \& Rincón, 2014; Spieza, 2010, entre otros). El uso de las TIC en el hogar es un factor positivo y significativo, ya que permite proveer a los estudiantes de las herramientas y las habilidades digitales requeridas en la escuela.

Al mismo tiempo, el efecto de las TIC en la escuela, medido a través del ratio de computadoras por estudiante, muestra un efecto significativo y positivo. Los alumnos que asisten a escuelas con una proporción mayor de computadoras por alumno alcanzan un mejor desempeño educativo en matemáticas.

Por otro lado, cabe señalar que el acceso a computadoras e Internet no es un indicador suficiente de uso de las TIC. Probablemente, los diferenciales en los patrones de uso con respecto al acceso sean mayores entre los hogares más pobres o con un estatus socioeconómico más bajo. Lamentablemente, no se dispone de datos en la Argentina sobre los patrones de uso en el hogar para corroborar esta hipótesis.

Asimismo, existen factores inobservables vinculados con el acceso a las TIC y al aprendizaje, tales como la actitud de los estudiantes hacia las TIC, habilidades, motivaciones, entre otros; que, si bien pretenden ser controlados con el método econométrico empleado, es casi imposible obtener resultados que contemplen a todos. Teniendo en cuenta esta limitación, se planea a futuro emplear metodologías alternativas como los modelos de ecuaciones estructurales SEM que permitan sobrellevar esto.

Desde el punto de vista de la política, los resultados sugieren la importancia de las acciones públicas dirigidas a difundir el acceso a las TIC, tanto en el hogar como en la escuela. El hallazgo de que el efecto de la disponibilidad 
de TIC en el hogar difiere entre escuelas implica que estas son disímiles en su gestión en relación con las diferencias que acarrean sus estudiantes desde sus posiciones iniciales y da lugar a la idea de que los establecimientos educativos son capaces de desarrollar estrategias en busca de disminuir la brecha tecnológica de origen. De este modo, se corroboran los programas existentes en la Argentina, como el Programa Conectar Igualdad, que acompañan la provisión de una computadora por estudiante en la escuela con su acceso en el hogar.

\section{Referencias}

Alderete, M. V., \& Formichella, M. M. (2015). Análisis del efecto de las TIC en el rendimiento educativo: el caso del Programa Conectar Igualdad en Argentina. Revista de la CEPAL 119, 89-107.

Angrist J., \& Lavy, V. (2002). New evidence on classroom computers and pupil learning. The Economic Journal 112, 735-765.

Aristizabal, G., Caicedo, M., \& Escandón D. (2009). Las Tecnologías de la Información y Comunicación como determinante en el rendimiento académico escolar, Colombia 2006-2009. Ponencia presentada en XXI Jornadas de la Asociación de Economía de la Educación, Oporto, Portugal. Recuperado de http:/ /2012.economicsofeducation.com

Aypay, A. (2010). Information and communication technology (ICT) usage and achivement of turkish students in PISA 2006. The Turkish Online Journal of Educational Technology, 9(2), 116-124.

Becta (2007). Inclusive Learning: an Essential Guide. Recuperado de http:/ / mirandanet.ac.uk/wp-content/uploads/2016/04/eg_inclusive_learning.pdf

Biagi, F., \& Loi, M. (2013). Measuring ICT Use and Learning Outcomes: evidence from recent econometric studies. European Journal of Education, 48(1), 28-42.

Botello, A. L. H., \& Rincón, G. A. (2014). La influencia de las Tic en el desempeño académico de los estudiantes en América Latina: Evidencia de la prueba PISA 2012. Lima: Memorias Virtual Educa, Memorias VE2014.

Buckingham, D. (2008). Más allá de la tecnología. Manantial, Buenos Aires CEPP (2008). Evaluación del Programa Todos los Chicos en la Red. Buenos Aires: Centro de Estudios en Políticas Públicas.

Calero, J., \& Escardibul, J. O. (2007). Evaluación de servicios educativos: el rendimiento en los centros públicos y privados medido en PISA-2003. Hacienda Pública Española, 183(4), 33-66.

Calero, J., Choi A., \& Waisgrais, S. (2009). Determinantes del rendimiento educativo del alumnado de origen nacional e inmigrante en PISA-2006. Cuadernos Económicos de ICE, 78, 281-310. 
Calero, J., Choi, A., \& Waisgrais, S. (2010). Determinantes del riesgo de fracaso escolar en España: una aproximación por medio de un análisis logístico multinivel aplicado a PISA-2006. Revista de Educación Número extraordinario, 2010, 225-256.

Carneiro, R., Toscano, J. C., \& Díaz, T. (2009). Los desafíos de las tic para el cambio educativo. Buenos Aires: Ed. OEI - Fundación Santillana.

Carrillo, P., Onofa, M., \& Ponce, J. (2010). Information Technology and Student Achievement: Evidence from a Randomized Experiment in Ecuador. IDB Working Paper 78. Recuperado de http:/ / www.iadb.org/res/publications / pubfiles/pubIDB-WP-223.pdf

Cervini, R. (1999). Factores asociados al logro escolar/5. Calidad y equidad en la educación básica en la Argentina. Buenos Aires: Ed. Ministerio de cultura y educación de la Nación.

Cervini, R. (2002). Desigualdades socioculturales en el aprendizaje de matemática y lengua de la educación secundaria en Argentina: un modelo de tres niveles. Revista Electrónica de Investigación y Evaluación Educativa (RELIEVE), 8(2), 135-158.

Cervini, R. (2003). Diferencias de resultados cognitivos y no-cognitivos entre estudiantes de escuelas públicas y privadas en la educación secundaria de Argentina: Un análisis multinivel. Archivos Analíticos de Políticas Educativas, 11(6), 1-32.

Cervini, R. (2012). El 'efecto escuela' en países de América Latina: reanalizando los datos del SERCE. Archivos Analíticos de Políticas Educativas, 20(39), 1-39.

Claro, M. (2011). El papel de las tecnologías de la información y las comunicaciones en la educación inclusiva. Santiago de Chile: Project Document, ECLAC.

Comber, C., Watling, R., Lawson, T., Cavendish, S., McEune, R., \& Paterson, F. (2002). ImpaCT2: Learning at home and school: Case studies. Coventry: Becta.

Cox, M., Webb, M., Abbott, C., Blakeley, B., Beauchamp, T., \& Rhodes, V. (2004). A review of the research literature relating to ICT and attainment. Londres: Becta.

Cristia, J., Ibarrarán, P., Cueto, S., Santiago, A., \& Severín, E. (2012). Technology and Child Development: Evidence from the One Laptop per Child Program.IDB Working Paper 304. Recuperado de http: / / www.iadb.org/ en/research-and-data

Decándido, G. (2011). Factores que afectan las competencias de los alumnos argentinos en PISA 2009. Un estudio empírico de dos niveles con efectos de interacción. Anales de la AAEP. Recuperado de http:/ / www.aaep.org.ar Diez-Roux A. (2002). A glossary for multilevel analysis. Journal of Epidemiology and Community Health, 56, 588-594. 
Formichella, M. M., Alderete, M. V., \& Di Meglio, G. A. (2015). El acceso a las TIC en el hogar como determinante del rendimiento educativo en el nivel medio: un análisis para Argentina. XXIV Jornadas de la Asociación de Economía de la Educación. Madrid.

Formichella, M. M. (2011) ¿Se debe el mayor rendimiento de las escuelas de gestión privada en la Argentina al tipo de administración? Revista de la CEPAL, 105, 151-166.

Formichella, M. M., \& Krüger, N. (2013). El fracaso escolar en el nivel medio argentino: ¿es menos frecuente en las escuelas de gestión privada debido a su administración? Regional and Sectoral Economic Studies, 13-3, 127-144.

Formichella, M. M., \& Ibáñez-Martin, M. M. (2014). Género e inequidad educativa: un análisis para el nivel medio en Argentina. Revista Regional and Sectoral Economic Studies/ Estudios Económicos Regionales y Sectoriales, 14-1, 196-210.

Hargittai, E. (2002). Second-level digital divide: difference in people's online skills. Recuperado de http:/ / firstmonday.org/htbin/cgiwrap/bin/ojs/ index.php/fm/article/view/942/864.

INDEC (2012). Encuesta Nacional sobre Acceso y Uso de Tecnologías de la Información y la Comunicación (ENTIC). Resultados del tercer trimestre de 2011.

Jewitt, C., \& Parashart, U. (2011). Technology and learning at home: findings for the evaluation of the Home access programme pilot. Journal of Computer Assisted Learning, 27, 303-313.

Kaztman, R. (2010). Impacto social de la incorporación de las nuevas tecnologías de información y comunicación en el sistema educativo. Serie Políticas Sociales 166. Santiago de Chile: Ed. CEPAL, Naciones Unidas.

Kozma, R. B. (2008). Comparative analysis of policies for ICT in education. En J. Voogt \& G. Knezek (eds.), International handbook on information technology in primary and secondary education (pp. 1.083-1.096). New York: Ed. Springe.

Kozma, R. B. (ed.) (2003). Technology, innovation and educational change: a global perspective. A Global Perspective: a Report of the Second Information Technology in Education Study, Module 2. Eugene, OR: Ed. International Society for Technology in Education (ITSE) Publications.

Krüger, N., \& Formichella, M. M. (2012). Escuela pública y privada en Argentina: una comparación de las condiciones de escolarización en el nivel medio. Revista Perspectivas: Revista de análisis de economía, comercio y negocios internacionales, 6(1), 113-144.

Kuhlemeier, H., \& Hemker, B. (2007). The impact of computer use at home on students' Internet skills. Computers $\mathcal{E}$ Education, 49, 460-480.

Lara, P., \& Duart, J. M. (2005). Gestión de contenidos en el e-learning: acceso y uso de objetos de información como recurso estratégico. Revista de Uni- 
versidad y Sociedad del Conocimiento, 2(2). Recuperado de http:/ / www. uoc.edu/rusc/2/2/dt/esp/lara.pdf

Machin, S., Mcnally, S., \& Silva, O. (2006). New technology in schools: is there a payoff? Economic Journal, 117, 1145-1167.

Maguire, C., \& Zhang, J. (2007). Blended learning in the development context. Experience with GDLN in Asia-Pacific, Tokyo Development Learning Center Japan/World Bank Distance Learning Partnership Project. Recuperado de https://www.jointokyo.org/files/cms/news/pdf/Blended_Learning_in_the_Development_Context_v1.pdf

Malamud, O., \& Pop-Eeches, C. (2010). Home computer and the development of human capital. NBER Working Paper 15814. Recuperado de www.nber. org/papers/w15814

Mediavilla, M., \& Escardíbul, J. O. (2015). El efecto de las TIC en la adquisición de competencias. Un análisis de género y titularidad de centro en las evaluaciones por ordenador. PISA 2012. Madrid: Informe Español.

Meelissen, R. M., \& Drent, M. (2008). Gender differences in computer attitudes: Does the school matter? Computers in Human Behavior, 24, 969-985.

Notten, N., \& Kraaykamp, G. (2009). Home media and science performance: a cross national study. Educational Research and Evaluation, 15, 367-384.

Notten, N., Peter, J., Kraaykamp, G., \& Valkenburg, P. M. (2009). Research note: digital divide across borders a cross-national study of adolescents' use of digital technologies. European Sociological Review, 25, 551-560.

OCDE (2003). PISA 2003. Manual de análisis de datos. Madrid: Ed. OCDE.

Parshall, C. G., Spray, J. A., Kalohn, J. C., \& Davey, T. (2002). Practical considerations in computer-based testing. New York: Ed. Springer.

Plowman, L., Stevenson, O., Pake, Mc., Stephen, C., \& Adey, C. (2011). Parents, pre-schoolers and learning with technology at home: some implications for policy. Journal of computer assisted learning, 27, 361-371.

Robinson, J. P, Di Maggio, P., \& Hargittai, E. (2003). New Social Survey Perspectives on the Digital Divide. IT E Society, 1(5), 1-22.

Rodriguez, P., Nussbaum, M., \& Dombrovskaia, L. (2013). ICT for education: a conceptual framework for the sustainable adoption of technology enhanced learning environments in schools. Technology, Pedagogy and Education, 21(3), 291-315.

Santos, M. (2007). Quality of Education in Argentina: Determinants and distribution using pisa 2000 test scores. Well-being and Social Policy. México D.F., México. Conferencia Interamericana de Seguridad Social y Universidad Iberoamericana, 3(1), 69-95. 
Spiezia, V. (2010). Does Computer Use Increase Educational Achievements? Student-level Evidence from PISA. OECD Journal: Economic Studies. Recuperado de http:/ / www1.oecd.org/eco/labour/49849896.pdf

Sunkel, G., \& Trucco, D. (2010). Nuevas Tecnologías de la información y la comunicación para la educación en América Latina: riesgos y oportunidades. Serie politicas sociales 167. Santiago de Chile: Ed. CEPAL, Naciones Unidas.

Sunkel, G., Trucco, D., \& Möller, S. (2011). Aprender y enseñar con tecnologías de la información y la comunicación (TIC) en América Latina. Potenciales beneficios. Serie Políticas Sociales 169. Santiago de Chile: Ed. ECLAC. Sunkel, G., \& Trucco, D. (Eds.).(2012). Las tecnologías digitales frente a los desafíos de una Educación Inclusiva en América Latina - Algunos casos de buenas prácticas. Santiago de Chile: Ed. Naciones Unidas.

Terzis, V., \& Economices, A. (2011). The acceptance and use of computer based assessment. Computers \& Education, 56, 1032-1044.

Volman, M., van Eck, E., Heemskerk, I., \& Kuiper, E. (2005). New technologies, new differences. Gender and ethnic differences in pupils use of ICT in primary and secondary education. Computers $\mathcal{E}$ Education, 45(1), 35-55.

Witte, K., \& Rogge, N. (2014). Does ICT matter for effectiveness and efficiency in mathematics education? Computers \& Education, 75, 173-184.

Wittwer, J., \& Senkbeil, M. (2008). Is students' computer use at home related to their mathematical performance at school? Computers \& Education, 50, 1558-1571. 